\title{
A Reason Maintenance Perspective on Relevant Ramsey Conditionals
}

\author{
Haythem O. Ismail, Department of Computer Science, German \\ University in Cairo, Cairo, Egypt.E-mail: haythem.ismail@guc.edu.eg
}

\begin{abstract}
This paper presents a Ramsey account of conditionals within the framework of an implemented reason maintenance system. The reason maintenance system is built on top of a deductive reasoning engine based on relevance logic. Thus, the account of conditionals provided is not susceptible to the fallacies of relevance. In addition, it is shown that independently motivated requirements on practical relevant reason maintenance allow us to gracefully circumvent Gärdenfors's triviality result.
\end{abstract}

Keywords: Conditionals; Ramsey test; Reason maintenance; Relevance logic

\section{Introduction}

Frank Ramsey's so called "Ramsey test" provides an intuitive link between conditionals (roughly, sentences of the form 'If $\mathrm{P}$ then Q') and belief change. The test grounds the plausibility of conditionals in a process of belief change. In an often quoted excerpt from [43], Robert Stalnaker gives a procedural interpretation of the Ramsey test:

First, add the antecedent (hypothetically) to your stock of beliefs; second, make whatever adjustments are required to maintain consistency (without modifying the hypothetical belief in the antecedent); finally, consider whether or not the consequent is then true.

This procedure is particularly appealing for researchers in artificial intelligence (AI), who have long been interested in reasoning about conditionals [5, 34, for example]. What makes it even more appealing is that, in its crucial second step, it provides a characterization of conditionals based on the (familiar to AI) concept of belief revision. The AI and philosophical literature on belief revision seem to have originated from different concerns. On one hand, the AI researchers were primarily motivated by the implementation issues of supplementing a general reasoning system with the facility to maintain consistency and revise its beliefs. This gave rise to what are known as "reason maintenance" (or "truth maintenance") systems. [8, 7, 32, for example]. On the other hand, the philosophers were keen to uncover a tight set of rationality postulates that govern the principles whereby a logical theory is to be revised [1]. These two attitudes have witnessed considerable convergence over the history of belief revision [33, 44, 21, for example].

To everyone's distress, however, Peter Gärdenfors [10] proved that the Ramsey test account of conditionals, together with some seemingly reasonable constraints on belief revision (three of the AGM postulates [1]), is inconsistent with a minimal set of harmless demands on a logical theory. Gärdenfors's result triggered considerable 


\section{A Reason Maintenance Perspective on Relevant Ramsey Conditionals}

research attempting to save the intuitive interpretation of conditionals provided by the Ramsey test $[11,37,14,4,28,13,12$, for example].

In this paper, I attempt to do two things. First, I will argue that, if belief revision is interpreted in the context of an implemented reason maintenance system, Gärdenfors's triviality result is avoided. This comes as a consequence of rejecting some of the assumptions made by Gärdenfors, based on general demands on implemented reason maintenance systems that are independent of conditionals and the triviality result. Second, as a side effect, I shall outline a theory for reasoning about conditionals within an implemented knowledge representation and reasoning (KRR) system with a reason maintenance component. The discussion will primarily focus on the SNePS KRR system [42, 40] and its reason maintenance component SNeBR [32, 20, 21]. The $\mathrm{SNePS}$ reasoning system is a natural deduction system of relevance logic [2]. Hence, the account of conditionals provided is not susceptible to the fallacies of relevance and, thus, avoids irrelevant conditionals along the lines of [31].

In Section 2, we review Gärdenfors's triviality theorem and, in Section 3, we examine previous attempts to rectify the damage it has wrought. Section 4 presents a reason maintenance system based on relevance logic, which is then extended to accommodate conditionals. This system is evaluated with respect to Gärdenfors's triviality result in Section 5. Finally, Section 6 provides an informal discussion of the status of iterated conditionals within our theory.

\section{The Triviality Trap}

What exactly did Gärdenfors discover? As pointed out in the introduction, he discovered that the Ramsey test is inconsistent with simple common demands on a logical system. In particular, Gärdenfors proved that the Ramsey test will introduce a contradiction into a belief set that contains none of three pair-wise contrary propositions. That such a belief set may exist is uncontroversial. Hence, the triviality result.

Nevertheless, Gärdenfors's proof is based on a mesh of background assumptions. These assumptions are primarily of two types: (i) assumptions on the belief revision process implied by the Ramsey test, and (ii) assumptions on what a "belief set" is. Attempts to circumvent the triviality result are based on dropping one or more of these assumptions. To get a deeper understanding of what exactly the problem is, and to appreciate previous approaches to solve it, we start by listing Gärdenfors's background assumptions. ${ }^{1}$

In what follows, $\mathcal{L}_{0}$ is a ground language of classical propositional or first-order logic including the absurd proposition $\perp^{2}, \mathcal{L}_{1}$ is the closure of $\mathcal{L}_{0}$ under the conditional connective $>, \mathbb{K}$ is the set of all belief sets, $\mathcal{K} \in \mathbb{K}$ is a belief set, and $*: \mathbb{K} \times \mathcal{L}_{1} \longrightarrow \mathbb{K}$ is a belief revision operator.

1. Assumptions on $\mathcal{K}$ :

$\left(A^{\mathcal{K}} 1\right)$ Belief sets are sets of sentences ${ }^{3}: \mathcal{K} \subseteq \mathcal{L}_{1}$.

$\left(A^{\mathcal{K}} 2\right)$ Belief sets may include conditional sentences: For some $\mathcal{K} \in \mathbb{K}, \mathcal{K} \nsubseteq \mathcal{L}_{0}$.

\footnotetext{
${ }^{1}$ This is not an exhaustive list. It is a list of those assumptions that are (so far) uncontroversial and/or relevant to my examination of previous work and my own proposal.

${ }^{2}$ It should be noted, however, that the triviality result was shown to be valid for a more general class of monotonic [38] and non-monotonic [29] logics.

${ }^{3}$ Gärdenfors [10] uses the term "proposition" instead of "sentence". Yet, he states that "belief sets are just theories in the standard logical sense" [10, p. 83].
} 
A Reason Maintenance Perspective on Relevant Ramsey Conditionals 3 $\left(A^{\mathcal{K}} 3\right)$ Belief sets are deductively-closed: if $\phi \in \mathrm{Cn}_{0}(\mathcal{K})$ then $\phi \in \mathcal{K} .{ }^{4}$

2. Assumptions on belief revision:

$\left(A^{*} 0\right)$ Closure of $\mathbb{K}$ under $*: \mathcal{K} * \phi \in \mathbb{K}$.

$\left(A^{*} 1\right)$ Success: $\phi \in \mathcal{K} * \phi$.

$\left(A^{*} 2\right)$ Consistency: If $\perp \in \mathrm{Cn}_{0}(\mathcal{K} * \phi)$ then $\perp \in \mathrm{Cn}_{0}(\{\phi\})$.

$\left(A^{*} 3\right)$ Expansion 1: $\mathrm{Cn}_{0}(\mathcal{K} * \phi) \subseteq \mathrm{Cn}_{0}(\mathcal{K} \cup\{\phi\})$.

$\left(A^{*} 4\right)$ Expansion 2: If $\neg \phi \notin \mathrm{Cn}_{0}(\mathcal{K})$, then $\mathrm{Cn}_{0}(\mathcal{K} \cup\{\phi\}) \subseteq \mathrm{Cn}_{0}(\mathcal{K} * \phi)$.

From $\left(A^{*} 4\right)$ and monotonicity of $\mathrm{Cn}_{0},\left(A^{*} 5\right)$ immediately follows:

$\left(A^{*} 5\right)$ Preservation: If $\neg \phi \notin \mathrm{Cn}_{0}(\mathcal{K})$, then $\mathrm{Cn}_{0}(\mathcal{K}) \subseteq \mathrm{Cn}_{0}(\mathcal{K} * \phi)$.

With these background assumptions, Gärdenfors [10] states the Ramsey test as follows.

$(R T) \phi>\psi \in \mathcal{K}$ if and only if $\psi \in \mathcal{K} * \phi$.

For ease of reference, let us break ( $R T)$ into two conditionals:

(RT1) If $\phi>\psi \in \mathcal{K}$ then $\psi \in \mathcal{K} * \phi$.

$(R T 2)$ If $\psi \in \mathcal{K} * \phi$ then $\phi>\psi \in \mathcal{K}$.

To simplify the proof of the triviality theorem, Gärdenfors first proves the following crucial lemma: The "monotonicity criterion".

$(M)$ For all $\mathcal{K}, \mathcal{K}^{\prime} \in \mathbb{K}$ and all $\phi \in \mathcal{L}_{1}$, if $\mathcal{K} \subseteq \mathcal{K}^{\prime}$ then $\mathcal{K} * \phi \subseteq \mathcal{K}^{\prime} * \phi$.

\section{Proof.}

$\begin{array}{cl}\text { 1. } \mathcal{K} \subseteq \mathcal{K}^{\prime} & \text { (Assumption) } \\ \text { 2. } \psi \in \mathcal{K} * \phi & \text { (Assumption) } \\ \text { 3. } \phi>\psi \in \mathcal{K} & (2,(R T 2)) \\ \text { 4. } \phi>\psi \in \mathcal{K}^{\prime} & (1,3) \\ \text { 5. } \psi \in \mathcal{K}^{\prime} * \phi & (4,(R T 1)) \\ \text { 6. } \mathcal{K} * \phi \subseteq \mathcal{K}^{\prime} * \phi & (2,5) \\ \text { 7. }(M) & (1,6)\end{array}$

Before we present Gärdenfors's triviality result, we define the notion of non-triviality of a belief revision system.

$(N T)$ A belief revision system $\left\langle\mathcal{L}_{1}, \mathbb{K}, *\right\rangle$ is non-trivial if, for some $\mathcal{K} \in \mathbb{K}$ and $A, B, C \in$ $\mathcal{L}_{1}$

1. $\{\neg(A \wedge B), \neg(A \wedge C), \neg(B \wedge C)\} \subseteq \mathrm{Cn}_{0}(\mathcal{K})$; and

2. $\neg A \notin \mathrm{Cn}_{0}(\mathcal{K}), \neg B \notin \mathrm{Cn}_{0}(\mathcal{K})$, and $\neg C \notin \mathrm{Cn}_{0}(\mathcal{K})$.

THEOREM 2.1

There is no non-trivial belief revision system that satisfies $\left(A^{\mathcal{K}} 1\right)-\left(A^{\mathcal{K}} 3\right),\left(A^{*} 1\right)-\left(A^{*} 4\right)$, and $R T$.

${ }^{4} \mathrm{Cn}_{0}$ is classical (deductive) logical consequence. We may assume a natural deduction system, although only modus ponens and the deduction theorem are needed. 
4 A Reason Maintenance Perspective on Relevant Ramsey Conditionals

\begin{tabular}{|l|c|c|c|c|c|c|c|}
\hline & $A^{\mathcal{K}} 1$ & $A^{\mathcal{K}} 2$ & $A^{\mathcal{K}} 3$ & $A^{*} 1$ & $A^{*} 2$ & $A^{*} 3$ & $A^{*} 4$ \\
\hline Rott [37] & & & & & & $\times$ & $\times$ \\
\hline Hansson [14] & & & $\times$ & & & $\times$ & $\times$ \\
\hline Arló Costa and Levi [4] & & $\times$ & & & & & \\
\hline Lindström and Rabinowicz [28] & $\times$ & & & & & & \\
\hline Grahne [13] & & & & & & & $\times$ \\
\hline Giordano et àl [12] & & & & & & $\times$ & $\times$ \\
\hline
\end{tabular}

TABLE 1: A comparison of six proposals to escape Gärdenfors's triviality result. The crosses indicate the culprits identified by each proposal.

\section{Proof.}

1. $\neg A \notin \mathcal{K}$

2. $\mathcal{K} * A=\mathrm{Cn}_{0}(\mathcal{K} \cup\{A\})$

3. $(B \vee C) \in(\mathcal{K} * A) *(B \vee C)$

4. $\neg(B \vee C) \notin(\mathcal{K} * A) *(B \vee C)$

5. $\neg C \notin(\mathcal{K} * A) *(B \vee C)$

6. $\neg(A \vee B) \notin \mathcal{K}$

7. $\mathcal{K} *(A \vee B)=\mathrm{Cn}_{0}(\mathcal{K} \cup\{A \vee B\})$

8. $\mathcal{K} *(A \vee B) \subseteq \mathcal{K} * A$

9. $(\mathcal{K} *(A \vee B)) *(B \vee C) \subseteq(\mathcal{K} * A) *(B \vee C)$

10. $\neg C \notin(\mathcal{K} *(A \vee B)) *(B \vee C)$

11. $\neg(B \vee C) \notin \mathcal{K} *(A \vee B)$

12. $(\mathcal{K} *(A \vee B)) *(B \vee C)=\mathrm{Cn}_{0}(\mathcal{K} *(A \vee B) \cup\{B \vee C\})$

13. $(\mathcal{K} *(A \vee B)) *(B \vee C)=\mathrm{Cn}_{0}(\mathcal{K} \cup\{A \vee B, B \vee C\})$

14. $(\mathcal{K} *(A \vee B)) *(B \vee C)=\mathrm{Cn}_{0}(\mathcal{K} \cup\{B\})$

15. $\neg C \in \mathrm{Cn}_{0}(\mathcal{K} \cup\{B\})$

16. $\neg C \in(\mathcal{K} *(A \vee B)) *(B \vee C)$

17. Contradiction

18. $\neg C \in(\mathcal{K} * A) *(B \vee C)$

19. $\neg B \in(\mathcal{K} * A) *(B \vee C)$

$(N T)$

$\left(1, A^{\mathcal{K}} 3, A^{*} 3, A^{*} 4\right)$

$\left(A^{*} 1\right)$

$\left(3, A^{*} 2\right)$

(Assumption)

$(N T)$

$\left(6, A^{\mathcal{K}} 3, A^{*} 3, A^{*} 4\right)$

$\left(2,7, A^{\mathcal{K}} 3, \mathrm{Cn}_{0}\right)$

$(8, M)$

$(5,9)$

$\left(7, N T, \mathrm{Cn}_{0}\right)$

$\left(11, A^{\mathcal{K}} 3, A^{*} 3, A^{*} 4\right)$

$(13, N T)$

$(N T)$

$\left(10,16, \mathrm{Cn}_{0}\right)$

20. Contradiction

(Similarly, 5-17)

$\left(4,18,19, \mathrm{Cn}_{0}\right)$

Having displayed the proof in detail, we can carefully analyze the different loopholes proposed in the literature. Each of the proposed loopholes identifies one or more of the background assumptions and/or the Ramsey test as the culprit.

\section{Loopholes}

We shall consider six proposals $[37,14,4,28,13,12]$, each identifying a different set of background assumptions as the culprit, or a different way out of the triviality trap. Except for [4], all proposals attempt to invalidate the use of $(M)$ in the proof. They all (pace Gärdenfors in [10,11]) preserve the Ramsey test (some version of it, for that matter), and choose to reject $M$ based on the background assumptions. Table 1 lists the culprits identified by each of the six proposals.

Rott [37] argues convincingly that $(M)$ is indeed true, but only trivially so. Infor- 
mally, once we admit conditionals into belief sets (as per $\left(A^{\mathcal{K}} 2\right)$ ), no belief set can be a proper subset of another. From this general result, the invalidity of $\left(A^{*} 3\right)$ and $\left(A^{*} 4\right)$ (and hence $\left(A^{*} 5\right)$ ) follows. The result is proved by Hansson [14], making use of the following property of proper subsets.

$(P S S) \mathcal{K} \in \mathbb{K}$ has a proper subset if and only if there are $\mathcal{K}_{1} \subseteq \mathcal{K}$ and $\mathcal{K}_{2} \subseteq \mathcal{K}$ such that $\mathcal{K}_{1} \nsubseteq \mathcal{K}_{2}$ and $\mathcal{K}_{2} \nsubseteq \mathcal{K}_{1}$.

To informally illustrate Hansson's proof, I will refer to an example based on one due to Darwiche and Pearl [6].

\section{Example 1.}

A murder occurs. John and Mary are the prime suspects. Detective 1 finds evidence incriminating John $\left(\mathcal{K}_{1}=\mathrm{Cn}_{0}(\{J\})\right)$. Detective 2, on the other hand, finds evidence incriminating Mary $\left(\mathcal{K}_{2}=\mathrm{Cn}_{0}(\{M\})\right)$. Both detectives report to their supervisor $\left(\mathcal{K}=\mathrm{Cn}_{0}(\{J, M\})\right)$. As long as we only consider sentences in $\mathcal{L}_{0}$, then it is clear that $\mathcal{K}_{1}, \mathcal{K}_{2}$ and $\mathcal{K}$ satisfy $(P S S)$. However, intuitively, $\neg(J \wedge M)>(J \wedge \neg M) \in \mathcal{K}_{1}{ }^{5}$ Similarly, $\neg(J \wedge M)>(\neg J \wedge M) \in \mathcal{K}_{2}$. But these two conditionals are contradictory; they cannot both be in $\mathcal{K}$.

The above example illustrates the fundamental difficulty in finding three sets satisfying $(P S S)$ : incomplete information licences the belief in conditionals that lose their support in a more informed belief state. Inspecting Table $1,[37,14,12]$ take issue with $\left(A^{*} 3\right)$ and $\left(A^{*} 4\right)$. Most probably $[37,14,12]$ would identify the main glitch in the triviality proof with step $7 .^{6}$ Though they agree on the culprit, each of these authors proposes a different way out of the triviality trap. Rott [37] informally considers employing a non-monotonic logic instead of $\mathrm{Cn}_{0}$. Hansson [14] presents a detailed theory founded on belief base revision. (Hence, his rejection of $\left(A^{\mathcal{K}_{2}}\right)$. $)^{7}$ Giordano et àl [12] restrict $\left(A^{*} 3\right)$ and $\left(A^{*} 4\right)$ to the maximal $\mathcal{L}_{0^{-}}$-subset of $\mathcal{K}$.

Grahne [13] would probably identify step 12 as the main glitch. Grahne's rejection of $\left(A^{*} 4\right)$ is based, not on admitting conditionals into belief states (as per $\left(A^{\mathcal{K}} 2\right)$ ), but on his very interpretation of the belief change operator appropriate for the interpretation of conditionals as per the Ramsey test. Instead of the classical AGM belief revision operator [1] , Grahne opts for Katsuno and Mendelzon's belief update operator [22]. Belief revision is appropriate for a change in belief signaled by acquiring information about a static world. Belief update, on the other hand, is needed when the change in belief is necessary due to a change in the world. When revising $\mathcal{K} *(A \vee B)$ with $B \vee C$ in step 12, we assume the world has not changed. That is, $A \vee B$ is still true. Thus, $B$ immediately follows, since adding $B \vee C$ just gives us more specific information about which of the contraries $A$ and $B$ is indeed true. On the other hand, when updating $\mathcal{K} *(A \vee B)$ with $B \vee C$, we assume that the world has changed, and, thus, cannot assume that $A \vee B$ is still true. To our best knowledge, only $B \vee C$ is certain, but not $B$.

Arló Costa and Levi [4] (following a hard-line position of Levi's [26]) reject $A^{\mathcal{K}} 2$.

\footnotetext{
${ }^{5}$ Hansson [14, p. 529] stresses that this last conclusion is based on "prephilosophical" intuitions, and (crucially) not on the Ramsey test.

${ }^{6}$ Of course, by rejecting $\left(A^{*} 3\right)$ and $\left(A^{*} 4\right)$, any step that is licensed by either is flawed. Nevertheless, those authors seem to base most of their informal arguments on the invalidity of step 7 .

${ }^{7} \mathrm{~A}$ belief base $\mathcal{B} \subseteq \mathcal{K}$ is a set of beliefs such that $\mathrm{Cn}_{0}(\mathcal{B})=\mathcal{K}$
} 


\section{A Reason Maintenance Perspective on Relevant Ramsey Conditionals}

They argue that conditionals do not qualify as members of belief sets (or as truth-value bearers), but as representations of an agent's dispositions to change their beliefs. From the premise that this is Ramsey's own position, Gärdenfors's $(R T)$ is disqualified as a formal rendering of the Ramsey test. Their proposal is to adopt a stratified theory, where there is a clear distinction between the belief set $\mathcal{K}$ (a subset of $\mathcal{L}_{0}$ ) and the set of sentences, $s(\mathcal{K}) \subseteq \mathcal{L}_{1}$, supported by $\mathcal{K}$. Only the latter may include conditionals. A stratified version of the Ramsey test may then be stated:

$(S R T)$ For all $\phi, \psi \in \mathcal{L}_{0}, \phi>\psi \in s(\mathcal{K})$ if and only if $\psi \in \mathcal{K} * \phi$.

Although a stratified version of $(M)$ may also be derived (with the antecedent being $s(\mathcal{K}) \subseteq s\left(\mathcal{K}^{\prime}\right)$ ), the proof of the triviality result will be blocked at step 8 , since $\mathcal{K} \subseteq \mathcal{K}^{\prime}$ does not entail $s(\mathcal{K}) \subseteq s\left(\mathcal{K}^{\prime}\right)$.

Lindström and Rabinowicz hold yet another position [28]. They identify $A^{\mathcal{K}} 1$ as the sole culprit, and, instead of taking $\mathcal{K}$ to be a set of sentences, they assume it is a set of propositions. How does this assumption get us out of the triviality trap? The assumption by itself may not help if sentences and propositions stand in one-to-one correspondence. This is exactly what Lindström and Rabinowicz reject. They argue that conditionals are context-sensitive: the same conditional may express different propositions in different contexts. ${ }^{8}$ On their account, a context is simply a belief set. ${ }^{9}$ By adopting a context-sensitive version of the Ramsey test, the proof of $(M)$ is blocked and the triviality result is avoided. Lindström and Rabinowicz's rendering of the Ramsey test could be presented as follows, where the semantics of $>$ depends on the context $\mathcal{K}$.

$(C R T) \phi>_{\mathcal{K}} \psi \in \mathcal{K}$ if and only if $\psi \in \mathcal{K} * \phi$.

The proof of $(M)$ blocks in step 5 , which cannot be proved since $>_{\mathcal{K}^{\prime}}$ is needed in place of $>_{\mathcal{K}}$ in step 4 .

In what follows, I will present an assumption-based reason maintenance system. The details of the system are based on assumptions that differ fundamentally from some of those underlying the triviality result. As it turns out, these assumptions, which are independently motivated by issues of rational agency and computational complexity, allow us to gracefully escape the triviality trap when the system is extended to accommodate conditionals.

\section{Reason Maintenance and Conditionals}

\subsection{General Requirements on Reason Maintenance}

Unlike belief revision theories, reason maintenance systems are required to take into account issues of bounded computational resources and availability. These issues motivate the following three requirements on reason maintenance systems.

RM1. Belief sets are not closed under logical consequence.

RM2. Paradoxes of implication are not tolerated.

\footnotetext{
${ }^{8}$ It should be noted that the same position was skeptically considered by Gärdenfors himself [10, p. 91].

${ }^{9}$ Here, I am using the term "belief set" to refer to sets of propositions. Lindström and Rabinowicz [28] use the term "belief state", instead; they reserve "belief set" for sets of sentences.
} 
RM3. Implicit inconsistencies are tolerated.

By "belief set" in RM1, we mean the contents of a knowledge base, representing the explicit beliefs of an agent. This is in contrast to the the notion of belief set found in the literature [1, for example], which refers to everything an agent is committed to believing. The motivation for RM1 is clear; no realistic computational (or rational) reasoning system can be logically closed. While we may talk about the closure of a belief set to facilitate the analysis of its potential theorems, the belief set itself must be finite, and as small as possible for that matter. Clearly, RM1 is at odds with $\left(A^{\mathcal{K}} 3\right)$.

RM2 is particularly required to block the derivation of arbitrary sentences from contradictions. From the point of view of rational agency, it is clear that agents (notably humans) can accommodate contradictory beliefs without committing to logical absurdity. From the point of view of computational reasoning systems, a system should provide useful, sound inferences even in the presence of contradictions.

RM3 is probably the least obvious. However, once RM2 is accepted, it is clear that the harmful effects of contradictions can be isolated. In addition, inconsistencies are only tolerated as long as they are only implicit. Once a contradiction is explicitly derived (that is, added to the belief set), then a process of consolidation is triggered, which results in restoring consistency by minimally evicting some of the beliefs responsible for the contradiction.

In what follows, a reason maintenance system that satisfies the above requirements will be presented. The system is based on [32] and [20, 21]. It is implemented as SNeBR, the belief revision component of the SNePS knowledge representation and reasoning system [42, 40]. Readers familiar with active logics [9, 3] will notice similarities in the motivations and assumptions underlying both active logics and SNePS logics. In particular, since its inception (as "step logic" [9]), active logic has always adopted RM1, RM2, and RM3. The details of the systems are different though. The main difference is that whereas the underlying logic in SNePS is a firstorder relevance logic [39], that of active logic is a special first-order logic where rules of inference are time-sensitive, allowing the system to reason about its own reasoning process as it evolves in time. This very feature, together with a form of reason maintenanc, provides active logics with the means of economically recovering from explicit contradictions. It should be noted, however, that a form of time-sensitivity has been built into some SNePS-based agents [19, 18, 41].

Before delving into the details of SNePS reason maintenance, we need to consider the relevance logic underlying SNePS reasoning.

\subsection{The System FR of Relevant Implication}

It is clear that the classical $\mathrm{Cn}_{0}$ does not observe RM2. SNePS logic is a version of Anderson and Belnap's relevance logic [2, 39]. Relevance logic was conceived as a result of the need to provide a notion of implication that does not suffer from the fallacies of relevance that the classical material implication exhibits. Hughes and Cresswell [17] refer to these as the "paradoxes of implication":

(1) $\phi \Rightarrow(\psi \Rightarrow \phi)$ 


\section{A Reason Maintenance Perspective on Relevant Ramsey Conditionals}

(2) $\neg \phi \Rightarrow(\phi \Rightarrow \psi)$

(1) states that a truth is implied by anything, and (2) states that a falsity implies anything. These two, together with the law of the excluded middle, entail the following intuitively-outrageous statement, which states that, given any two propositions, one of them implies the other.

$(3)(\phi \Rightarrow \psi) \vee(\psi \Rightarrow \phi)$

The system $F R$ of Anderson and Belnap [2] is a natural deduction system, with two rules (of elimination and introduction) for each logical connective (and quantifier, in case of a quantified logic). As usual, a proof in this system is a sequence of formulas and other proofs (sub-proofs). The first item in a proof may be an arbitrary formula considered as an hypothesis. Every other formula in the proof has to follow from previous items as a result of applying the rules of inference. The major difference between the system $F R$ and classical natural deduction systems is that every formula that appears in a proof is associated with a label. Labels in [2] were considered to be sets of natural numbers. Here, we will take them to be sets of formulas of the underlying language $\mathcal{L}$. The rules of inference are sensitive to these labels. Here are some examples of these rules of inference, where $P$ is an arbitrary proof and $P(i)$ is the $i^{\text {th }}$ step of $P$, which is either a formula or a sub-proof (a full exposition may be found in [39]):

(HYP) $P(1)$ may be a formula $\phi$ with label $\{\phi\}$.

$(\Rightarrow E$ ) For $i>2, P(i)$ may be a formula $\psi$ with label $\alpha \cup \beta$, provided that $P(j)$ is $\phi$ with label $\alpha$ and $P(k)$ is $\phi \Rightarrow \psi$ with label $\beta$ for some $j, k<i$.

$(\Rightarrow I)$ For $i>1, P(i)$ may be a formula $\phi \Rightarrow \psi$ with label $\alpha \backslash\{\phi\}$, provided that, for some $j<i, P(j)$ is a sub-proof $P_{2}$, where $P_{2}(1)$ is the formula $\phi$ with label $\{\phi\}$ and $P_{2}(k)$ (for some $k$ ) is $\psi$ with label $\alpha$ such that $\phi \in \alpha$.

$(\vee E)$ For $i>2, P(i)$ may be a formula $\psi$ with label $\alpha \cup \beta$, provided that $P(j)$ is $\phi \vee \psi$ with label $\alpha$ and $P(k)$ is $\neg \phi$ with label $\beta$ for some $j, k<i$.

$(\vee I)$ For $i>2, P(i)$ may be a formula $\phi \vee \psi$ with label $\alpha$, if there is a formula $\xi$ such that, for some $j, k<i, P(j)$ is $\xi \Rightarrow \phi$ with label $\alpha$ and $P(k)$ is $\neg \xi \Rightarrow \psi$ with label $\beta$.

The requirement that $\phi$ be a member of $\alpha$ in $(\Rightarrow I)$ is the distinguishing feature of relevant implication: $\phi$ has to have been used in deriving $\psi$ in order for $\phi \Rightarrow \psi$ to follow. Similarly, the classical rule of $\vee$-introduction is replaced by the above version, where a disjunction is only asserted if the disjuncts are relevant to one another (via a formula $\xi$ as indicated in $(\vee I)$ above, together with the law of the excluded middle). ${ }^{10}$ Similar elimination and introduction rules are introduced for other logical connectives. These rules manipulate the labels to secure a notion of relevant entailment.

In addition to the elimination and introduction rules, there is a rule of re-iteration. This rule depends on the notion of a context function that assigns to every natural number (corresponding to a step in a proof) a set of labelled formulas.

\footnotetext{
${ }^{10}$ Actually, the system $F R$ of $[2]$ accepts classical $\vee$-introduction and rejects $\vee E$. The effect is the same for the
} purposes of this paper, however. 
DEFINITION 4.1

A context function $\mathcal{C}_{P}: \mathbb{N} \longrightarrow \mathcal{L} \times 2^{L}$ of a proof $P$ assigns to every step $i$ in $P$ the set of labelled formulas $\mathcal{C}_{P}(i)=\left\{\langle P(j), \alpha\rangle \mid P(j) \in \mathcal{L}_{0}\right.$ is a formula with label $\alpha$ and $j<i\}$

$(R I)$ For every $i$ and $j$, if $P(i)$ is a sub-proof $P_{2}$, then $P_{2}(j)$ may be a formula $\phi$ with label $\alpha$, provided that $\langle\phi, \alpha\rangle \in \mathcal{C}_{P}(i)$.

Thus, FR follows the the common scoping convention, where formulas of a superproof are visible inside a sub-proof, so long as they appear before the sub-proof. That is, all results derived in a super-proof, so far, are available to a sub-proof.

For example, following is a proof of the theorem $(P \Rightarrow Q) \Rightarrow((Q \Rightarrow R) \Rightarrow(P \Rightarrow$ $R)$ ). Each line of the proof starts by a dotted sequence of integers whose length indicates the depth of sub-proof nesting, and whose elements indicate the step number within each nested sub-proof.

$\begin{array}{ll}\text { 1.1. }\langle P \Rightarrow Q,\{P \Rightarrow Q\}\rangle & (\mathrm{HYP}) \\ \text { 1.2.1. }\langle Q \Rightarrow R,\{Q \Rightarrow R\}\rangle & \text { (HYP) } \\ \text { 1.2.2. }\langle P \Rightarrow Q,\{P \Rightarrow Q\}\rangle & (R I) \\ \text { 1.2.3.1. }\langle P,\{P\}\rangle & (\mathrm{HYP}) \\ \text { 1.2.3.2. }\langle P \Rightarrow Q,\{P \Rightarrow Q\}\rangle & (R I) \\ \text { 1.2.3.3. }\langle Q \Rightarrow R,\{Q \Rightarrow R\}\rangle & (R I) \\ \text { 1.2.3.4. }\langle Q,\{P, P \Rightarrow Q\}\rangle & (\Rightarrow E) \\ \text { 1.2.3.5. }\langle R,\{P, P \Rightarrow Q, Q \Rightarrow R\}\rangle & (\Rightarrow E) \\ \text { 1.2.4. }\langle P \Rightarrow R,\{P \Rightarrow Q, Q \Rightarrow R\}\rangle & (\Rightarrow I) \\ 1.3 .\langle(Q \Rightarrow R) \Rightarrow(P \Rightarrow R),\{P \Rightarrow Q\}\rangle & (\Rightarrow I) \\ \text { 2. }\langle(P \Rightarrow Q) \Rightarrow((Q \Rightarrow R) \Rightarrow(P \Rightarrow R)),\{\}\rangle & (\Rightarrow I)\end{array}$

We conclude this section by pointing out that relevance logic ensures relevance by keeping track of the history of derivations. Thus, we seem to have an independent motivation for recording derivation traces, which is required by assumption-based reason maintenance. This being said, we now turn to the reason maintenance component of our system.

\subsection{The Case of $\mathcal{L}_{0}$}

First, let us consider a reason maintenance system for the non-conditional language $\mathcal{L}_{0}$. In what follows, $\mathrm{Cn}_{R}$ denotes relevance logic consequence.

DEFINITION 4.2

A support set of a sentence $\phi \in \mathcal{L}_{0}$ is a set $s \subseteq \mathcal{L}_{0}$ such that $\phi \in \operatorname{Cn}_{R}(s)$. $s$ is relevantly minimal if, for every $\psi \in s, \psi \Rightarrow \phi \in \mathrm{Cn}_{R}(s \backslash\{\psi\})$.

Relevantly minimal support sets of a sentence $\phi$ are akin to Hansson's $\phi$-kernels [15], but with a slight twist. Hansson's $\phi$-kernels are support sets that are subsetminimal-no proper subset of a $\phi$-kernel is a support set of $\phi$. This is sufficient but not necessary for relevant minimality. A relevantly minimal support set $s$ includes all and only those sentences that are relevant to an actually discovered derivation of $\phi$. There might be shorter derivations, from a proper subset of $\phi$, that are, nevertheless, yet to be discovered. 


\section{A Reason Maintenance Perspective on Relevant Ramsey Conditionals}

DEFINITION 4.3

A belief state $\mathcal{S}$ is a quadruple $\langle\mathcal{K}, \mathcal{B}, \sigma, \preccurlyeq\rangle$, where:

1. $\mathcal{K} \subseteq \mathcal{L}_{0}$ is a belief set.

2. $\mathcal{B} \subseteq \mathcal{K}$, with $\mathcal{K} \subseteq \operatorname{Cn}_{R}(\mathcal{B})$, is a belief base. If $\phi \in \mathcal{B}$, then $\phi$ is a base belief.

3. $\sigma: \mathcal{K} \longrightarrow 2^{2^{\mathcal{B}}}$ is a support function, where each $s \in \sigma(\phi)$ is a relevantly minimal support set of $\phi$. If $\phi \in \mathcal{B}$, then $\{\phi\} \in \sigma(\phi)$.

4. $\preccurlyeq \subseteq \mathcal{B} \times \mathcal{B}$ is a total pre-order on base beliefs. ${ }^{11}$

On the intuitive interpretation of the above definition, base beliefs are beliefs that have independent standing. For example, they are the result of perception or interaction with another agent (possibly a human operator/user). Crucially, they are not in the belief state based solely on inference. Note that no constraints are imposed on the complexity of base beliefs. In particular, they need not be atomic in any syntactic sense (cf. [24]). The belief set $\mathcal{K}$ is not closed under $\mathrm{Cn}_{R}$; it represents the set of sentences that are either base beliefs or that were actually derived from base beliefs. ${ }^{12}$ This is in contrast to the logically-closed $\mathrm{Cn}_{R}(\mathcal{K})$ which is the set of sentences derivable from base beliefs.

The set $\sigma(\phi)$ is the family of minimal support sets that were actually (relevantly) used, or discovered, to derive $\phi . \mathcal{B}$ may include minimal support sets of $\phi$ that are, nevertheless, not in $\sigma(\phi)$, if they are not yet discovered to derive $\phi$. The total preorder $\preccurlyeq$ represents a preference ordering over base beliefs. This ordering will be used when belief revision requires sacrificing a base belief; the least preferred will be the victim. I will refrain from making any commitments about the origins of this ordering. In particular, unlike standard epistemic entrenchment relations [16, for example], I am not assuming any logical basis for preference. For the purpose of this paper, the ordering is just given. ${ }^{13}$

The rules of inference presented in Section 4.2 may be restated in terms of belief states. A belief state can be thought of as a snapshot of an ever-evolving proof. ${ }^{14}$ Every time a base belief is introduced, it is introduced as the hypothesis of a new sub-proof, where all older formulas are re-iterated. The set of supports, $\sigma$, is a generalization of labels to sets of sets of formulas (that is, to sets of labels as per Section 4.2). The formulation of the rules will have to be slightly adjusted to accommodate this difference.

Belief revision in this system is a distant variant of Hansson's semi-revision [16] - a non-prioritized belief revision operator where success is not guaranteed. We first need to define a notion of relevant expansion.

\section{DEFINITION 4.4}

Let $\mathcal{S}=\langle\mathcal{K}, \mathcal{B}, \sigma, \preccurlyeq\rangle$ be a belief state. A relevant expansion of $\mathcal{S}$ with $\phi \in \mathcal{L}_{0}$ is a belief state $\mathcal{S}+\phi=\left\langle\mathcal{K}_{+\phi}, \mathcal{B}_{+\phi}, \sigma_{+\phi}, \preccurlyeq+\phi\right\rangle$, with the following properties:

$\left(A^{+} 1\right)$ Success: $\mathcal{B}_{+\phi}=\mathcal{B} \cup\{\phi\}$.

$\left(A^{+} 2\right)$ Relevant inclusion: $\mathcal{K}_{+\phi}=\mathcal{K} \cup\left\{\psi \mid \phi \Rightarrow \psi \in \operatorname{Cn}_{R}(\mathcal{K})\right\}$.

\footnotetext{
${ }^{11} \mathrm{~A}$ total pre-order is a complete, reflexive, and transitive binary relation.

12 Thus, in time, a belief state can evolve into a different belief state that share the same base.

${ }^{13}$ For future investigation, we may consider the possibility of moving $\preccurlyeq$ into the object language.

${ }^{14}$ On how the non-monotonicity effect of belief revision fits this picture, the reader should check the discussion of filtering functions in Section 4.4
} 
$\left(A^{+} 3\right)$ Relevant Support: If $\psi \in \mathcal{K}_{+\phi}$ and $s \in \sigma_{+\phi}(\psi)$ then either $\psi \in \mathcal{K}$ and $s \in \sigma(\psi)$, or there is $s^{\prime} \in \sigma_{+\phi}(\phi)$ such that $s^{\prime} \subseteq s$.

$\left(A^{+} 4\right)$ Order preservation: $\preccurlyeq+\phi$ is the smallest total pre-order on $\mathcal{B}_{+\phi}$ satisfying $\preccurlyeq \subseteq \preccurlyeq+\phi$.

The belief state resulting from relevant expansion by $\phi$ will include $\phi$ and anything that follows from it. That all newly derived sentences indeed follow from $\phi$ is guaranteed by $\left(A^{+} 2\right)$ and $\left(A^{+} 3\right)$. In addition, old sentences may acquire new support only as a result of discovered derivations from $\phi\left(\left(A^{+} 3\right)\right)$. It should be noted that, given certain constraints on the implementation of $\mathrm{Cn}_{R}$, the set $\mathcal{K}_{+\phi}$ may be guaranteed to be finite (provided that $\mathcal{K}$ is). $\left(A^{+} 4\right)$ makes the simplifying assumption that adding $\phi$ does not disturb the preference relations already established; $\phi$ simply gets added in some appropriate position in the $\preccurlyeq$-induced chain of equivalence classes.

One way to implement relevant expansion is simply as assertion with forward inference. This is indeed the intended interpretation. However, as defined, relevant expansion admits other possibilities. For example, all the above properties will be satisfied if all newly-derived propositions are actually derived, not from $\phi$ (though, in principle, they are derivable from $\phi$ as per $\left.\left(A^{+} 2\right)\right)$, but from base beliefs that support $\phi$. This can happen if $\phi$ is already in $\mathcal{K}$ (and not self-supported). We can actually strengthen the definition of + to admit only the intended interpretation. However, this will unnecessarily complicate the exposition. As it turns out, this rather loose definition of + suffices for our purposes.

Based on relevant expansion, we can define a relevant version of revision.

\section{DEFINITION 4.5}

Let $\mathcal{S}=\langle\mathcal{K}, \mathcal{B}, \sigma, \preccurlyeq\rangle$ be a belief state. A relevant revision of $\mathcal{S}$ with $\phi \in \mathcal{L}_{0}$ is a belief state $\mathcal{S} \dot{+} \phi=\left\langle\mathcal{K}_{\dot{+} \phi}, \mathcal{B}_{\dot{+} \phi}, \sigma_{\dot{+} \phi}, \preccurlyeq \dot{+} \phi\right\rangle$, with the following properties:

$\left(A^{\dot{+}} 1\right)$ Base inclusion: $\mathcal{B}_{\dot{+} \phi} \subseteq \mathcal{B}_{+\phi}$.

$\left(A^{\dot{+}} 2\right)$ Inclusion: $\mathcal{K}_{\dot{+} \phi} \subseteq \mathcal{K}_{+\phi}$.

$\left(A^{\dot{+}} 3\right)$ Lumping: $\psi \in \mathcal{K}_{+\phi} \backslash \mathcal{K}_{\dot{+} \phi}$ if and only if, for every $s \in \sigma_{+\phi}(\psi), s \nsubseteq \mathcal{B}_{\dot{+} \phi}$.

$\left(A^{\dot{+}} 4\right)$ Preferential core-retainment: $\psi \in \mathcal{B}_{+\phi} \backslash \mathcal{B}_{\dot{+} \phi}$ if and only if $\perp \in \mathcal{K}_{+\phi}$ and $\psi \in$ $\left\{\xi \mid \exists s \in \sigma_{+\phi}(\perp), \xi \in s\right.$, and $\left.\forall \zeta \in s, \xi \preccurlyeq+\phi \zeta\right\}$.

$\left(A^{\dot{+}} 5\right)$ Support update: If $\psi \in \mathcal{K}_{\dot{+} \phi}$, then $\sigma_{\dot{+} \phi}(\psi)$ is the largest subset of $\sigma_{+\phi}(\psi)$ with range restricted to sets of subsets of $\mathcal{B}_{\dot{+} \phi}$.

$\left(A^{\dot{+}} 6\right)$ Order preservation: $\preccurlyeq \dot{+} \phi$ is the restriction of $\preccurlyeq+\phi$ to $\mathcal{B}_{\dot{+} \phi}$.

Thus, relevant revision is assertion with forward inference followed by consolidation [16]. Consolidation may be implemented by removing least-preferred beliefs (as per $\preccurlyeq)$ from each support set of $\perp$ in the inconsistent belief state resulting from expansion by $\phi$. (See the operation of kernel consolidation in [21].) As a result of consolidation, some base beliefs might be retracted in case relevant expansion with $\phi$ results in a contradiction. ${ }^{15}\left(A^{\dot{+}} 1\right)$ captures this intuition. Since belief sets are not the logical closures of their bases, $\left(A^{\dot{+}} 2\right)$ does not necessarily follow from $\left(A^{\dot{+}} 1\right)$. It is needed to indicate that relevant revision does not result in derivations that are not accounted for

${ }^{15}$ Technically, the contradiction need not be supported by $\phi$. However, in practice, a reason maintenance system should not tolerate explicit contradiction (SNeBR does not). Thus, prior to relevant revision with $\phi$, we may assume that no explicit contradiction was around, and, thus, that $\phi$ is somehow responsible for discovering/introducing a contradiction. 


\section{A Reason Maintenance Perspective on Relevant Ramsey Conditionals}

by relevant expansion. $\left(A^{+} 3\right)$ makes sure that only sentence that are still supported are believable. ${ }^{16}$

$\left(A^{\dot{+}} 4\right)$ guarantees that base beliefs that are evicted to retain (explicit) consistency indeed must be evicted. In addition, if a choice is possible, base beliefs that are least preferred are chosen for eviction. Note that, according to the above definition, this selection strategy is skeptical; that is, if multiple least preferred beliefs exist, all are evicted. This strategy, however, is only adopted here to simplify the exposition, and nothing relevant depends on it. ${ }^{17}$

As a simple corollary, it follows from $\left(A^{\dot{+}} 3\right)$ and $\left(A^{\dot{+}} 4\right)$ that the resulting belief state is not known to be inconsistent:

$\left(A^{\dot{+} 7}\right)$ Contradiction ignorance: $\perp \notin \mathcal{K}_{\dot{+} \phi}$

\subsection{The Case of $\mathcal{L}_{1}$}

To extend the reason maintenance system presented above to $\mathcal{L}_{1}$, a number of superficial alterations of the definitions are needed. The important point, however, is to devise an extension of $\mathrm{Cn}_{R}$ that accommodates conditionals. Adding the connective $>$ to the language, we need two inference rules - one for elimination and one for introduction. First, a piece of notation.

DEFINITION 4.6

Let $\mathcal{S}=\langle\mathcal{K}, \mathcal{B}, \sigma, \preccurlyeq\rangle$ be a belief state. The hypothetical expansion of $\mathcal{S}$ with $\phi \in \mathcal{L}_{1}$ is a belief state $\mathcal{S} \mp \phi=\left\langle\mathcal{K}_{\mp \phi}, \mathcal{B}_{\mp \phi}, \sigma_{\mp \phi}, \preccurlyeq \mp \phi\right\rangle$ where

1. $\mathcal{B}_{\mp \phi}=\mathcal{B} \cup\{\phi\}$

2. $\mathcal{K}_{\mp \phi}=\mathcal{K} \cup\{\phi\} ;$

3. $\sigma_{\mp \phi}(\phi)=\{\{\phi\}\}$ and $\sigma_{\mp \phi}(\psi)=\sigma(\psi)$ for $\psi \neq \phi$; and

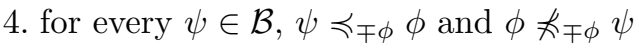

Hypothetical expansion (re)introduces $\phi$ into the belief state with independent standing as a most preferred belief. It is similar to the do operator of Pearl [35] in that it detaches $\phi$ from its derivational history (causal history, in the case of Pearl). We now define the elimination and introduction rules for $>$ as follows.

$(>E)$ If $\phi, \phi>\psi \in \mathcal{K}$, then $\psi$ may be added to $\mathcal{K}$, with $\sigma(\psi)=\left\{s_{\phi} \cup s_{>} \mid\left\langle s_{\phi}, s_{>}\right\rangle \in\right.$ $\sigma(\phi) \times \sigma(\phi>\psi)\}$.

$(>I)$ If $\psi \in\left(\mathcal{K}_{\mp \phi}\right)_{\dot{+} \phi}$, then $\phi>\psi$ may be added to $\mathcal{K}$ provided that $\sigma(\phi>\psi)=$ $\left\{s \backslash\{\phi\} \mid s \in\left(\sigma_{\mp \phi}\right) \dot{+} \phi(\psi)\right.$ and $\left.\phi \in s\right\}$ is not empty.

The elimination rule $(>E)$ is a direct extension of Anderson and Belnap's rule $(\Rightarrow E)$ for the elimination of relevant implication (see Section 4.2). The introduction rule $(>I)$ is also an extension of $(\Rightarrow I)$. The extension in this case is, by no means, direct though. $(>I)$ is actually the right-to-left direction of the Ramsey test $(R T 2)$, within the context of relevance logic. In simple English, the rule describes a procedure whereby one may decide whether to believe in the conditional $\phi>\psi$ :

\footnotetext{
${ }^{16}$ By "lumping", I'm referring to the lumping relation of Kratzer [25], whereby certain propositions either stay together or go together.

${ }^{17} \mathrm{Well}$, not exactly. If we would like to account for might-counterfactuals [25], then maybe the selection strategy would become crucial.
} 
1. Hypothetically expand the belief state with $\phi$.

2. Perform relevant forward inference to derive all sentences that could be derived from $\phi$.

3. Consolidate the resulting belief state, giving $\phi$ highest preference.

4. If $\psi$ is in the resulting belief state, accept $\phi>\psi$.

In addition to deciding on whether to accept $\phi>\psi$, we also compute its support sets along the way. The relevance of this derivation is guaranteed by the procedure used to compute $\sigma(\phi>\psi)$ : We only consider support sets that were added as a result of relevant expansion with $\phi$. The hypothetical expansion step allows us to readily distinguish those support sets since $\phi$ is necessarily a member of each. This eliminates cases where a conditional is only accepted as a result of its consequent being already in the belief set. The final removal of $\phi$ from the sets of supports is inherited from the rule $(\Rightarrow I)$ of Section 4.2 .

Note that step 1 in the above procedure uses hypothetical expansion rather than relevant revision. There are two reasons for this. First, given the definition of $\preccurlyeq \mp \phi$, hypothetically expanding with $\phi$, unlike relevant revision, guarantees success. This seems to be a requirement of any belief change operator appropriate for the Ramsey account of counterfactuals. Second, decoupling $\phi$ from its derivation history, as per the definition of $\sigma_{\mp \phi}(\phi)$, guarantees the correct computation of the set of supports of $\phi>\psi$ according to $(>I)$, as indicated above. In particular, it is possible that $\phi$ is already supported in the initial belief state. It is also possible that a belief supporting $\phi$ will also support a derivation of $\psi$ from $\phi$. Such a supporting belief should be included in a set of supports of $\phi>\psi$. Making $\sigma_{\mp \phi}(\phi)$ oblivious of this belief prevents its accidental removal from $\sigma(\phi>\psi)$.

Restricting the scope of admissible conditionals to only relevant ones allows us to reject examples like (4) and (5) as reported in [31]:

(4) If someone were to create a square circle, then we would not be amazed.

(5) If someone were to scare a pregnant guinea pig, then all her babies will be born without tails.

In most accounts of conditionals [43, 27, 24, 25, 34, for example] (but notably not in [31]), (4) and (5) will come out as acceptable counterfactuals. Assuming that axioms of geometry hold in all possible worlds, the first will be trivially acceptable since the antecedent is contradictory. The second will be accepted since the consequent is true in all worlds, closest to the real world, in which the antecedent is true. Interestingly, this is due to the irrelevance of the antecedent to the consequent. ${ }^{18}$ In our system, on the other hand, both (4) and (5) will not be accepted, since the set of supports of $\psi$ indicated in $(>I)$ will be empty.

In terms of proofs and sub-proofs as per Section 4.2 , both $(>I)$ and $(\Rightarrow I)$ may be considered as special cases of a more general rule of conditional-introduction. The trick is to slightly generalize the rule $(R I)$ of re-iteration. The generalization requires every sub-proof to declare a filter.

${ }^{18}$ Or maybe, if one were to force themselves to accept (5), then a relevance link will naturally be assumed. 


\section{A Reason Maintenance Perspective on Relevant Ramsey Conditionals}

DEFINITION 4.7

A filtering function $\mathcal{F}_{P}: \mathcal{L}_{1} \times 2^{\mathcal{L}_{1}} \longrightarrow \mathcal{L}_{1} \times 2^{\mathcal{L}_{1}}$ of a proof $P$ assigns to every set $A$ of labelled formulas a subset thereof.

We can now generalize $(R I)$ thus:

(GRI) For every $i$ and $j$, if $P(i)$ is a sub-proof $P_{2}$, then $P_{2}(j)$ may be a formula $\phi$ with label $\alpha$, provided that $\langle\phi, \alpha\rangle \in \mathcal{F}_{P_{2}}\left(\mathcal{C}_{P}(i)\right)$.

Now, a generalized rule of conditional-introduction will look exactly like $(\Rightarrow I)$. However, depending on what the sub-proof $\left(P_{2}\right)$ is intended for, its filtering function will look different. For $\Rightarrow$, the filtering function will filter-in all members of $\mathcal{C}_{P}(\mathrm{i})$. (In terms of belief states, all elements of $\mathcal{K}$ are available for the sub-proof.) On the other extreme, if the filtering function filters-in none of the members of $\mathcal{C}_{P}(\mathrm{i})$, we get a rule of strict-implication introduction.

The rule $(>I)$ is just the special case that emerges when $\mathcal{F}_{P_{2}}$ filters-in only those members of $\mathcal{C}_{P}(i)$ that do not contradict $P_{2}$ 's hypothesis, as selected by relevant revision. Whether other possible filtering functions will prove useful is a matter for future investigation. ${ }^{19}$

By adding $(>E)$ and $(>I)$ to our repertoire of inference rules, we define an extension $\mathrm{Cn}_{R}>$ of $\mathrm{Cn}_{R}$ for relevant conditional consequence. All the definitions of Section 4.3 may now be extended to $\mathcal{L}_{1}$ by replacing each occurrence of $\mathcal{L}_{0}$ by $\mathcal{L}_{1}$, and each occurrence of $\mathrm{Cn}_{R}$ by $\mathrm{Cn}_{R>}$. This may be considered overly permissive by many scholars. For, now, we allow two things that are traditionally not allowed.

We allow, pace Hansson [14], (i) belief bases to include conditional sentences and (ii) belief states to be revised with conditional sentences. The justification for this is the same: we view conditionals to possibly have independent standing. For a rational agent or knowledge representation and reasoning system, this is actually reasonable. The following example of a "useful counterfactual" is due to Costello and McCarthy $[5$, p. 1].

(6) If another car had come over the hill when you passed that car, there would have been a head-on collision

A natural context in which (6) may be uttered is one in which the speaker is teaching someone an important safety rule of car driving. Most probably, the person receiving this utterance does not have enough experience to have concluded it themselves. The person can learn from (6), though. And they can do that without ever trying to achieve its antecedents. Thus, it seems that in similar cases, the only reasonable thing to assume is that the conditional is a base belief that probably could not have been derived without external help.

The above notwithstanding, the reader might still be suspicious about this permissive attitude towards conditionals. This point will be considered in detail in the following sections.

\section{The Trap Reentered}

What can we say now about the Ramsey test and Gärdenfors's triviality result? First of all, any step in the proof that depends on closure $\left(A^{\mathcal{K}} 3\right)$, success $\left(A^{*} 1\right)$,

\footnotetext{
${ }^{19}$ For readers familiar with [24], our filtering functions seem to have a striking similarity to Kratzer's "partition
} functions". 
or consistency $\left(A^{*} 2\right)$ will be invalid, given that these assumptions are not condoned by our system. In addition, even with closure, success, and consistency reinstated, we will show below that the system avoids the result on several grounds. Notably, it encapsulates variants of the solutions proposed in $[37,14,28]$. In addition, the triviality trap is also avoided as a result of adopting relevance logic and only one direction of the Ramsey test.

\subsection{The Uni-Directional Ramsey Test}

As $(>I)$ indicates, only one direction of the Ramsey test $(R T 2)$ is adopted; we use it as a rule of introduction for conditionals. Lacking the other direction $(R T 1)$, the proof of monotonicity $(M)$ will be blocked, and the triviality result may thus be avoided. But let us consider whether the exclusion of $(R T 1)$ is justified.

A direct reversal of $(>I)$ would yield.

$(R R T 1)$ If $\phi>\psi$ may be added to $\mathcal{K}$ with

$$
\sigma(\phi>\psi)=\left\{s \backslash\{\phi\} \mid s \in\left(\sigma_{\mp \phi}\right)_{\dot{+} \phi}(\psi) \text { and } \phi \in s\right\},
$$

then $\psi \in\left(\mathcal{K}_{\mp \phi}\right)_{\dot{+} \phi}$ and $\left\{s \backslash\{\phi\} \mid s \in\left(\sigma_{\mp \phi}\right)_{\dot{+} \phi}(\psi)\right.$ and $\left.\phi \in s\right\}$ is not empty.

This does not look right. For what does it mean to say that $\phi>\psi$ may be added to $\mathcal{K}$ ? Note that it does not mean that $\phi>\psi$ is in $\mathcal{K}$, for $\mathcal{K}$ is not logically closed. The primary way, within our system, we can make sure that $\phi>\psi$ may be added to $\mathcal{K}$ is probably by using $(>I)$. But, then, $(R R T 1)$ will not be very useful; it is only telling us something that we already know about $\mathrm{Cn}_{R>}$.

So maybe we can simply replace "may be added to $\mathcal{K}$ " with "is in $\mathcal{K}$ ". But, then, the first antecedent $(\phi>\psi \in \mathcal{K})$ would imply the second consequent $(\sigma(\phi>\psi) \neq \varnothing)$, and the second antecedent (the definition of $\sigma(\phi>\psi)$ ) would imply the first consequent $\left(\psi \in\left(\mathcal{K}_{\mp \phi}\right)_{\dot{+} \phi}\right)$. Again, this version of $R R T 1$ does not seem useful.

Finally, we can simply drop all mention of supports and state that

(7) if $\phi>\psi \in \mathcal{K}$, then $\psi \in\left(\mathcal{K}_{\mp \phi}\right) \dot{+} \phi$.

This is far from being the converse of $(>I)$. But let us consider what it says. $(7)$ is the closest we can get in our system to $(R T 1)$. It claims that the only way one may come to believe in (or accept) a conditional $\phi>\psi$ is through reasoning. That is, to accept $\phi>\psi$, it actually has to follow from what the person already knows. Is this reasonable?

As pointed out in Section 4.4, one may come to believe a conditional as a result of its being told to them (by some reliable source); witness (6), for example. Requiring one to be able to derive the conditional themselves is too extreme. For example, it means that fifth graders cannot do matrix arithmetic until they study advanced linear algebra, and forever memorize (or comprehend) all the knowledge required to generate relevant proofs.

Moreover, it seems that conditionals are most useful in exactly those cases where they cannot be independently derived. For, if I have enough knowledge to derive a conditional (via $(R T 2)$ or the equivalent $(>I)$ ), then deriving the conditional, at best, saves me some future reasoning steps through the use of $(>E)$. On the other 


\section{A Reason Maintenance Perspective on Relevant Ramsey Conditionals}

hand, if I cannot independently derive $\phi>\psi$, this will be due to lack of knowledge. Accepting the conditional will, in this case, allow me to make useful inferences, despite the (sometimes unfortunate) fact that I do not know exactly how relevant $\phi$ is to $\psi$. What I will know for sure is that the world, albeit not completely accessible to me, is such that some relation of relevance links $\phi$ to $\psi$. My reliable source may be aware of this relation, and maybe they are not. Maybe they are as clueless as I am, but they accept the conditional out of trust in yet another source. Eventually, someone has to have arrived at the conditional through reasoning via $(>I)$. But, crucially, this someone need not be me.

Another way to interpret (7) is to consider the status of $\phi>\psi$ in the belief state resulting from revision with $\phi$. If $\phi>\psi$ is in this belief state, then, surely through $(>E), \psi$ will also be there. However, if $\phi>\psi$ is not in the $\phi$-revised belief state, then nothing may be concluded about the status of $\psi$. Thus, perhaps all what (7) claims is that $\phi>\psi$, by all means, survives revision with $\phi$. Now, I do not see how useful this may be, at least from the point of view of rational agency or a KRR system. If anything, it may save us some time by caching one of the results of hypothetically revising with $\phi$ (we can also easily reconstruct the supports of $\psi$ in the resulting belief state).

This, however, is not unproblematic. For, in general, $\phi>\psi$ need not survive revision with $\phi$. Considering the following variant of Example 1 may clarify this point (where $\phi=M$ and $\psi=\neg J$ ). ${ }^{20}$

Example 2. The supervisor believes (probably by default) that only one person committed the murder: $\{J>\neg M, M>\neg J\}$. Detective 1 reports evidence incriminating John. This results in adding the beliefs $J$ and $\neg M$ to the supervisor's belief set. Now detective 2 reports evidence incriminating Mary. At this point, the supervisor needs to do some consolidation. Assuming that the evidence provided by both detectives is highly reliable, the supervisor would disbelieve $J>\neg M$, rendering $\neg M$ no longer supported. In addition, note that $M>\neg J$ also needs to be removed, to block the derivation of $\neg J$. Thus, when faced with strong evidence to the contrary, the supervisor gives up the belief in a single murderer: $M>\neg J$ does not survive revision with $M$.

\subsection{The Factor of Relevance}

The move from $\mathrm{Cn}_{0}$ to $\mathrm{Cn}_{R>}$ as the underlying logic turns out to have a devastating effect on the triviality result. First, note that step 8 of the proof of Theorem 2.1 is obviously invalid. For, even if we admit closure, $\mathrm{Cn}_{R>}(\mathcal{K} \cup\{A \vee B\}) \nsubseteq \mathrm{Cn}_{R>}(\mathcal{K} \cup\{A\})$. This follows from the fact $A \vee B \notin \mathrm{Cn}_{R>}(\{A\})$, for arbitrary $A$ and $B$ (see Section $4.2)$.

But someone might suspect that this is just a happy coincidence that relies on the particular formulation of the proof and the non-triviality condition presented here. The suspicion is justified, but (fortunately) not valid. In fact, the factor of relevance blocks the proof of monotonicity, which is needed for any version of the triviality result. (In particular, I shall also consider the version presented in [38], which is supposed to be a generalization of the one in [10].)

\footnotetext{
${ }^{20}$ Also see the discussion of the same point in the final section of [28].
} 
For ease of reference, here is a regeneration of the proof of monotonicity $(M)$ :
1. $\mathcal{K} \subseteq \mathcal{K}^{\prime}$
(Assumption)
2. $\psi \in \mathcal{K} * \phi$
(Assumption)
3. $\phi>\psi \in \mathcal{K}$
$(2,(R T 2))$
4. $\phi>\psi \in \mathcal{K}^{\prime}$
$(1,3)$
5. $\psi \in \mathcal{K}^{\prime} * \phi$
$(4,(R T 1))$
6. $\mathcal{K} * \phi \subseteq \mathcal{K}^{\prime} * \phi$
7. $(M)$

The problem in this proof is the move from step (2) to step (3). For, in order to introduce $\phi>\psi$, not only does $\psi$ have to be a member of $\mathcal{K} * \phi$, it also needs to relevantly follow from $\phi$. This is the gist of $(>I)$. Thus, the move from step (2) to step (3) is not valid for an arbitrary member $\psi \in \mathcal{K} * \phi$. Hence, even if we assume some version of $(R T 1)$, we cannot conclude the inclusion of $\mathcal{K} * \phi$ in $\mathcal{K}^{\prime} * \phi$.

As pointed out above, the proof of Theorem 2.1 presented in Section 2 will be blocked in step 8 . Now, we may try to patch the proof so that it still goes through. The only valid alternative to step 8 is

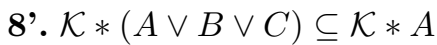

Here, $A \vee B \vee C$ may be relevantly derived (using ( $\vee I$ ) from Section 4.2), given $(N T)$. But, if we then replace all occurrences of $\mathcal{K} *(A \vee B)$ with $\mathcal{K} *(A \vee B \vee C)$, the move from step 13 to step 14 will no longer be valid.

Now, could it be that we are only able to block the proof due to its dependence on the classical $\vee$-introduction rule, and that this dependence is just an artifact of the particular formulation of the triviality result presented in [10]? To make sure that it is not, we consider the generalized argument presented by Krister Segerberg in [38]. Based on the assumptions presented in Section 2, and a slightly different notion of non-triviality, Segerberg argues as follows:

1. There are three propositions $A_{0}, A_{1}$, and $B$ such that $B$ is consistent with $A_{0}$ and $A_{1}$ taken separately, but not with their conjunction. (This is Segerberg's version of non-triviality.)

2. By $\left(A^{*} 1\right)$ and $\left(A^{*} 4\right), A_{0}, B \in\left\{A_{0}\right\} * B$.

3. Similarly, $A_{1}, B \in\left\{A_{1}\right\} * B$.

4. By monotonicity, $A_{0}, A_{1}, B \in\left\{A_{0}, A_{1}\right\} * B$

5. This means that $\left\{A_{0}, A_{1}\right\} * B$ is inconsistent, which violates $\left(A^{*} 2\right)$.

Once monotonicity is rejected as pointed out above, the argument does not go through. But perhaps one can adopt a weakened version of monotonicity, which is nevertheless valid under $\mathrm{Cn}_{R}$ :

$(R M)$ For all $\mathcal{K}, \mathcal{K}^{\prime} \in \mathbb{K}$ and all $\phi, \psi \in \mathcal{L}_{1}$, if $\mathcal{K} \subseteq \mathcal{K}^{\prime}$ and $\phi \Rightarrow \psi \in \mathcal{K} * \phi$ then $\phi \Rightarrow \psi \in \mathcal{K}^{\prime} * \phi$.

The proof of $(R M)$ is straightforward, assuming some version of $(R T 1)$. Can this version of monotonicity support Segerberg' argument? The only way it can is if

1. $B \Rightarrow A_{0} \in\left\{A_{0}\right\} * B$, and, hence $A_{0} \in\left\{A_{0}\right\} * B$; and 


\section{A Reason Maintenance Perspective on Relevant Ramsey Conditionals}

2. $B \Rightarrow A_{1} \in\left\{A_{1}\right\} * B$, and, hence $A_{1} \in\left\{A_{1}\right\} * B$

But, if $B \Rightarrow A_{1}$, then $B$ is inconsistent with $A_{0}$. Similarly, if $B \Rightarrow A_{0}$, then $B$ is inconsistent with $A_{1}$. This contradicts the non-triviality assumption. Thus, given non-triviality, the proof will never go through.

\subsection{Context Sensitivity}

In [28], Lindström and Rabinowicz pointed out the context-sensitive nature of conditionals. In our system, context-sensitivity is manifested in several ways. In particular, the acceptance of a conditional in a belief state $\mathcal{S}=\langle\mathcal{K}, \mathcal{B}, \sigma, \preccurlyeq\rangle$, not only depends on the contents of $\mathcal{K}$, but also on how $\mathcal{K}$ evolved into its current structure. That is, the acceptance of a conditional depends also on $\mathcal{B}, \sigma$, and $\preccurlyeq$.

Hansson [14, p. 531-532] argued that even if two belief sets are identical, baserevising them may yield different results. Thus, any step in the proof of Theorem 2.1 that relies on the equality of revising two identical belief sets will be invalid (for example, step 13).

To illustrate this point, I will allow myself to slightly modify an example by Hansson $[14$, p. 531-532] in order to accommodate the factor of relevance.

Example 3. Let $\mathcal{S}_{1}$ be a belief state with $\mathcal{B}_{1}=\{p \Leftrightarrow q\}$. Consider, $\mathcal{S}_{2}=\mathcal{S}_{1} \dot{+} p$ and $\mathcal{S}_{3}=\mathcal{S}_{1} \dot{+} q$. Clearly, $\mathcal{B}_{2}=\{p, p \Leftrightarrow q\}, \mathcal{B}_{3}=\{q, p \Leftrightarrow q\}$, and $\operatorname{Cn}_{R>}\left(\mathcal{B}_{2}\right)=\operatorname{Cn}_{R>}\left(\mathcal{B}_{3}\right)$. In particular, if $q \in \mathcal{K}_{2}$, then $\sigma_{2}(q)=\{\{p, p \Leftrightarrow q\}\}$. Similarly, if $p \in \mathcal{K}_{3}$, then $\sigma_{3}(p)=\{\{q, p \Leftrightarrow q\}\} .{ }^{21}$ Now consider $\mathcal{S}_{2} \dot{+} \neg p$ and $\mathcal{S}_{3} \dot{\dot{ }} \neg p$, and assume that $p \preccurlyeq \neg p$ in $\mathcal{S}_{2}$ and $p \Leftrightarrow q \preccurlyeq q$ in $\mathcal{S}_{3}$. Given Definition $4.5, q$ is in the second belief set and $\neg q$ is derivable in the first.

Thus, if two people hold the same set of beliefs, which nevertheless originates from different sets of premises along with different preference criteria, they are apt to revise their beliefs differently. ${ }^{22}$

Finally, similar to [28], the proof of $(M)$ will be blocked at step 5:

$$
\begin{array}{ll}
\text { 1. } \mathcal{K} \subseteq \mathcal{K}^{\prime} & \text { (Assumption) } \\
\text { 2. } \psi \in\left(\mathcal{K}_{\mp \phi}\right)_{\dot{+} \phi} \text { and }\left\{s \backslash\{\phi\} \mid s \in\left(\sigma_{\mp \phi}\right)_{\dot{+} \phi}(\psi) \text { and } \phi \in s\right\} \neq \varnothing & \text { (Assumption) } \\
\text { 3. } \phi>\psi \in \mathcal{K} & (2,(>I)) \\
\text { with } \sigma(\phi>\psi)=\left\{s \backslash\{\phi\} \mid s \in\left(\sigma_{\mp \phi}\right)_{\dot{+} \phi}(\psi) \text { and } \phi \in s\right\} & \\
\text { 4. } \phi>\psi \in \mathcal{K}^{\prime} & \\
\text { with } \sigma^{\prime}(\phi>\psi)=\left\{s \backslash\{\phi\} \mid s \in\left(\sigma_{\mp \phi}\right)_{\dot{+} \phi}(\psi) \text { and } \phi \in s\right\} & (1,3)
\end{array}
$$

At this point we are stuck; we cannot prove $\psi \in\left(\mathcal{K}_{\mp \phi}^{\prime}\right)_{\dot{+} \phi}$ since this requires $\sigma^{\prime}$, and not $\sigma$, in the definition of the support of $\phi>\psi$. For instance, in Example 2, where $\mathcal{K}=\{J>\neg M, M>\neg J\}$ and $\mathcal{K}^{\prime}=\mathcal{K} \cup\{J\}$, it is clear that the set of supports

\footnotetext{
${ }^{21}$ The "if" is needed since $\mathcal{K}$ is not necessarily the closure of $\mathcal{B}$

${ }^{22}$ For example, consider Quine's famous case of Bizet and Verdi [36], where we are asked to judge the acceptability of the following two conditionals.

(a) If Bizet and Verdi were fellow countrymen, then Verdi would be French.

(b) If Bizet and Verdi were fellow countrymen, then Bizet would be Italian.

Two agents with identical belief sets, including beliefs that Bizet was French and that Verdi was Italian, may judge (a) and (b) differently based on their preference criteria. For example, an agent that prefers Bizet's being French to Verdi's being Italian would most likely accept (a) and reject (b).
} 
of $M>\neg J$ in $\left(\mathcal{K}_{\mp M}\right)_{\dot{+} M}$ is different from that in $\left(\mathcal{K}_{\mp M}^{\prime}\right)_{\dot{+} M}$, where in the former it is simply $\{\{M>\neg J\}\}$ and in the latter it is the empty sets.

\section{Iterated Conditionals}

Before concluding, I guess at least some informal remarks should be made about iterated conditionals $[26,23]$. In Section 4.4, it was pointed out that the current system allows revision with conditional sentences. Allowing conditional revision means that the system can adequately account for iterated conditionals. This is indeed possible, provided we accept the attitude toward conditionals presented in Section 5.1 .

A general iterated conditional has the form

$$
(\phi>\psi)>(\xi>\zeta)
$$

This gives rise to two special cases whose analysis should suffice, where $\phi, \xi \in \mathcal{L}_{0}$ :

(8) $\phi>(\xi>\zeta)$

(9) $(\phi>\psi)>\xi$

Typically, conditionals of the form (8) are unproblematic. To introduce (8), first revise your beliefs with $\phi$. The conditional should then be introduced if $\xi>\zeta$ follows (recursively) from the resulting belief state. This is exactly what $(>I)$ and $\left(A^{+} 2\right)$ from Definition 4.4 yield. ${ }^{23}$

Conditionals of the form (9) are notoriously the problematic ones. For, in this case, we need to first adjust the belief state to one where $\phi>\psi$ is accepted, and then we can backward chain on $\xi$. The problem is that, in theories that accept both directions of the Ramsey test (primarily $(R T 1)$ ), the adjustment of the belief state to accommodate a conditional generally requires both retraction and expansion. In such theories, $\psi$ has to follow from $\phi$ in the adjusted belief state, which mandates equipping the adjusted state with sufficient knowledge for the derivation to work. As usual, this should be done with minimal damage to the current belief state. Expansion with minimal damage, however, is not as well-focused as retraction with minimal damage. For one can make potentially infinitely-many assumptions that would provide a relevant link from $\phi$ to $\psi$.

By rejecting the left-to-right direction $(R T 1)$ of the Ramsey test (see Section 5.1), we seem to be in a better position. For, now, we do not have to worry about which assumptions we should make for the derivation of $\phi>\psi$ to go through. We are already happy to accept $\phi>\psi$ without being able to derive it. Thus, we only need to worry about what to retract in order to make sure that the resulting belief state is consistent with $\phi>\psi$.

Clearly, we should retract formulas of the form $\neg(\phi>\psi)$ or $\phi>\neg \psi$. Such formulas may not be readily in $\mathcal{K}$, but may still follow from it. If we add the rule that $(\phi>$ $\neg \psi) \Rightarrow \neg(\phi>\psi)$, then, again, the current system will yield the desired effect.

The assertion-with-forward-inference interpretation of + would reveal formulas of the form $\neg(\phi>\psi)$ if they do follow from $\mathcal{K}$. However, traditional forward chaining

\footnotetext{
${ }^{23}$ But we may have to give up the simple assertion-with-forward-inference interpretation of + , or modify $(>I)$
} to include an element of backward chaining on $\psi$. 


\section{A Reason Maintenance Perspective on Relevant Ramsey Conditionals}

will not uncover beliefs of the form $\phi>\neg \psi$. But $+\phi$ may be redefined as forward chaining on $\phi$, followed by backward chaining on $\neg \phi$. The two definitions will have the same effect if $\phi \in \mathcal{L}_{0}$. If $\phi$ is a conditional, however, backward chaining will put the full power of $(>I)$ at our disposal. Formulas of the form $\phi>\neg \psi$ will be uncovered and retracted in the course of revision with $\phi>\psi$.

\section{Conclusions}

Based on [32, 21], I have presented a reason maintenance system with an underlying relevance logic. I have shown how such a system could be extended to accommodate a relevance-logical account of conditionals. One direction of the Ramsey test is used as a conditional-introduction inference rule. This rule hints at a promising account of iterated conditionals.

Given independently motivated assumptions on the underlying logic, the belief state, and the belief revision operator, Gärdenfors's triviality result is avoided. In particular, employing relevance logic proves to be sufficient for blocking the notorious monotonicity property that underlies various incarnations of the triviality result. In addition, by leaving out the left-to-right direction of the Ramsey test, we are, again, able to block monotonicity, and to simplify accounts of iterated conditionals.

The context-sensitivity of conditionals is accounted for by the infra-structure comprised of a belief base, a support function, and a preference ordering underlying a belief set. Similar to [28], this context-sensitivity provides yet another loophole out of the triviality trap.

The system presented here is similar to that of [14] in its use of base revision. It is different, however, in allowing conditional sentences to be members of belief bases and candidates for expansion and revision. Compared to the system of [13], the revisionbased approach presented here is another practical alternative to the update-based approach of [13]. It is my conviction that, ultimately, both revision and update are needed for reasoning about conditionals. In particular, the relation between indicative conditionals and belief revision on one hand, and subjunctive conditionals and belief update on the other hand, remains to be investigated.

Another point for future investigation is a semantic account of the theory of conditionals presented here. A possible starting point is the work of Mares and Fuhrmann on relevant conditionals [31]. Their account, however, is truth conditional. An adequate semantics of the conditionals presented here will have to be agent-oriented, in the sense that it should account for the notion of accepting conditionals based on reliable sources, which themselves are other agents. Another promising direction is based on Kratzer's work reported in [25]. Given its situation semantics, this account of conditionals may be more compatible with the notion of incomplete knowledge stressed in our system. In addition, situation semantics has also been proposed as a semantics of relevance logic [30]. Combining both approaches may prove adequate for the theory of the relevant conditionals presented here.

\section{Acknowledgements}

The author thanks two anonymous reviewers for their insightful comments and critical remarks on the penultimate version of the paper. Many thanks go to Christoph 
Beierle, Gabriele Kern-Isberner, and other participants of the KI-07 workshop on dynamics of knowledge and belief for discussions of an earlier draft of the paper.

\section{References}

[1] Carlos E. Alchourron, Peter Gärdenfors, and David Makinson. On the logic of theory change: Partial meet contraction and revision functions. The Journal of Symbolic Logic, 50(2):510-530, June 1985.

[2] Alan Anderson and Nuel Belnap. Entailment, volume I. Princeton University Press, Princeton, NJ, 1975.

[3] Michael L. Anderson and Donald R. Perlis. Logic, self-awareness and self-improvement: The metacognitive loop and the problem of brittleness. Journal of Logic and Computation, 15(1):2140, 2005.

[4] Horacio Arló Costa and Isaac Levi. Two notions of epistemic validity: Epistemic models for Ramsey's conditionals. Synthese, 109:217-262, 1996.

[5] Tom Costello and John McCarthy. Useful counterfactuals. Linköping Electronic Articles in Computer and Information Science, 4(12), 1999.

[6] Adnan Darwiche and Judea Pearl. On the logic of iterated belief revision. Artificial Intelligence, 89:1-29, 1997.

[7] Johan de Kleer. An assumption-based TMS. Artificial Intelligence, 28:127-162, 1986.

[8] Jon Doyle. A truth maintenance system. Artificial Intelligence, 12(3):231-272, 1979.

[9] Jennifer J. Elgot-Drapkin and Donald Perlis. Reasoning situated in time I: Basic concepts. Journal of Experimental and Theoretical Artificial Intelligence, 2:75-98, 1990.

[10] Peter Gärdenfors. Belief revisions and the Ramsey test for conditionals. The Philosophical Review, 95(1):81-93, 1986.

[11] Peter Gärdenfors. Variations on the Ramsey test: More triviality results. Studia Logica, 46:321$327,1987$.

[12] Laura Giordano, Valentina Gliozzi, and Nicola Olivetti. Belief revision and the Ramsey test: A solution. In $A I^{*} I A$ 2001: Advances in Artificial Intelligence : 7th Congress of the Italian Association for Artificial Intelligence, number 2175 in LNAI, pages 165-175, Berlin/Heidelberg, 2001. Springer-Verlag.

[13] Gösta Grahne. Updates and counterfactuals. Journal of Logic and Computation, 8(1):87-117, 1998.

[14] Sven Ove Hansson. In defence of the Ramsey test. The Journal of Philosophy, 89(10):522-540, October 1992.

[15] Sven Ove Hansson. Kernel contraction. Journal of Symbolic Logic, 59(3):845-859, September 1994.

[16] Sven Ove Hansson. A survey of non-prioritized belief revision. Erkenntnis, 50(2-3):413-427, 1999.

[17] G. E. Hughes and M. J. Cresswell. A New Introduction to Modal Logic. Routledge, London and New York, 1996.

[18] Haythem O. Ismail. Reasoning and Acting in Time. PhD thesis, University at Buffalo, The State University of New York, 2001.

[19] Haythem O. Ismail and Stuart C. Shapiro. Two problems with reasoning and acting in time. In Anthony Cohn, Fausto Giunchiglia, and Bart Selman, editors, Principles of Knowledge Representation and Reasoning: Proceedings of the Seventh International Conference (KR 2000), pages 355-365, San Francisco, CA, 2000. Morgan Kaufmann.

[20] Frances L. Johnson and Stuart C. Shapiro. Automatic belief revision in SNePS. In C. Baral and M. Truszczynski, editors, Proceedings of the 8th International Workshop on Non-Monotonic Reasoning (NMR2000), 2000. Unpaginated, 5 pages.

[21] Frances L. Johnson and Stuart C. Shapiro. Dependency-directed reconsideration: Belief base optimization for truth maintenance systems. In Proceedings of the Twentieth National Conference on Artificial Intelligence (AAAI-05), pages 313-320, 2005. 


\section{A Reason Maintenance Perspective on Relevant Ramsey Conditionals}

[22] Hirofumi Katsuno and Alberto Mendelzon. On the difference between updating a knowledge base and revising it. In Principles of Knowledge Representation and Reasoning: Proceedings of the second International Conference (KR'91), pages 387-394, 1991.

[23] Gabriele Kern-Isberner. Postulates for conditional belief revision. In Thomas Dean, editor, Proceedings of the Sixteenth International Joint Conference on Artificial Intelligence (IJCAI99), pages 186-191. Morgan Kaufmann, 1999.

[24] Angelika Kratzer. Partition and revision: The semantics of counterfactuals. Journal of Philosophical Logic, 10:201-216, 1981.

[25] Angelika Kratzer. An investigation into the lumps of thougth. Linguistics and Philosophy, 12:607-653, 1989.

[26] Isaac Levi. Iteration of conditionals and the Ramsey test. Synthese, 76:49-81, 1988.

[27] David Lewis. Counterfactuals. Blackwell Publishers, Oxford, England, 1973.

[28] Sten Lindström and Wlodzimierz Rabinowicz. The Ramsey test revisited. In G. Grocco, L. Fariñas del Cerro, and A. Herzig, editors, Conditionals: From philosophy to computer science, pages 147-191. Oxford University Press, Oxford, UK, 1996.

[29] David Makinson. The Gärdenfors impossibility theorem in non-monotonic contexts. Studia Logica, 49(1):1-6, 1990.

[30] Edwin Mares. Relvant logic and the theory of information. Synthese, 109:345-360, 1997.

[31] Edwin Mares and André Fuhrmann. A relevant theory of conditionals. Journal of Philosophical Logic, 24:645-665, 1995.

[32] João Martins and Stuart C. Shapiro. A model for belief revision. Artificial Intelligence, 35(1):25$79,1988$.

[33] Bernhard Nebel. A knowledge level analysis of belief revision. In Ronald Brachman, Hector Levesque, and Raymond Reiter, editors, Principles of Knowledge Representation and Reasoning: Proceedings of the First International Conference (KR'89), pages 301-311, Toronto, ON, 1989. Morgan Kaufmann.

[34] Charles Ortiz. Explanatory update theory: Applications of counterfactual reasoning to causation. Artificial Intelligence, 108(1-2):125-178, 1999

[35] Judea Pearl. Causality. Cambridge University Press, New York, 2000.

[36] Willard Van Orman Quine. Methods of Logic (Fourth Edition). Harvard University Press, Cambridge, Massachusetts, 2006.

[37] Hans Rott. Conditionals and theory change: Revisions, expansions, and additions. Synthese, $81: 91-113,1989$.

[38] Krister Segerberg. A note on an impossibility theorem of Gärdenfors. Noûs, 23(3):351-354, June 1989.

[39] Stuart C. Shapiro. Relevance logic in computer science. In Alan Anderson, Nuel Belnap, and M Dunn, editors, Entailment, volume II, pages 553-563. Princeton University Press, Princeton, NJ, 1992.

[40] Stuart C. Shapiro. SNePS: A logic for natural language understanding and commonsense reasoning. In Łucja M. Iwańska and Stuart C. Shapiro, editors, Natural Language Processing and Knowledge Representation: Language for Knowledge and Knowledge for Language, pages 175-195. AAAI Press/The MIT Press, Menlo Park, CA, 2000.

[41] Stuart C. Shapiro and Haythem O. Ismail. Anchoring in a grounded layered architecture with integrated reasoning. Robotics and Autonomous Systems, 43(2-3):97-108, 2003.

[42] Stuart C. Shapiro and William J. Rapaport. SNePS considered as a fully intensional propositional semantic network. In N. Cercone and G. McCalla, editors, The Knowledge Frontier, pages 263-315. Springer-Verlag, New York, 1987.

[43] Robert Stalnaker. A theory of conditionals. In Nicholas Rescher, editor, Studies in Logical Theory, number 2 in American Philosophical Quarterly, Monograph Series, pages 98-112. Basil Blackwell Publishers, Oxford, 1968.

[44] Mary-Anne Williams. Transmutations of knowledge systems. In J. Doyle, E. Sandewall, and P. Torasso, editors, Principles of Knowledge Representation and Reasoning: Proceedings of the Fourth International Conference (KR'94), pages 619-629, San Francisco, CA, 1994. Morgan Kaufmann.

Received December 1, 2007 